\title{
Advances in Interventional Neuroimaging
}

\author{
Vallabh Janardhan and Adnan I. Qureshi \\ Departments of Neurology, Neurosurgery, and Radiology, and Zeenat Qureshi Stroke Research Center, University of Minnesota, \\ Minneapolis, Minnesota 55455
}

\begin{abstract}
Summary: Advances in neuroimaging have aided in the development of useful diagnostic modalities for neurological disease and have helped improve existing therapies and pioneer newer therapies in the field of interventional neurology, a new subspecialty of neurology. In this chapter, the authors discuss the advances in various neuroimaging modalities, such as digital subtraction angiography, transcranial and intravascular ultrasonography, interventional dynamic computed tomography and 64-slice computed tomographic
\end{abstract}

scanners, magnetic resonance image-guided interventions and intravascular magnetic resonance imaging, and molecular and cellular neuroimaging tools using microbubbles. The authors also summarize the clinical usefulness of these advances and their role in improving the diagnostic and therapeutic potential of neurointerventional procedures. Key Words: Interventional neurology, interventional neuroradiology, endovascular neurosurgery, neuroimaging, angiography, intravascular ultrasonography.

\section{INTRODUCTION}

Interventional neurology and neuroimaging are relatively new subspecialties of neurology that use minimally invasive technology that includes catheter-based interventions, neuroimaging, and clinical neurological expertise to diagnose and treat diseases of the CNS, head, neck and spine. This chapter summarizes some of the recent advances in neuroimaging modalities that have helped advance the diagnostic and therapeutic potential of these cutting-edge neurointerventional procedures.

\section{ADVANCES IN INTERVENTIONAL NEUROIMAGING}

\section{Digital subtraction angiography based advances}

Flat panel detectors. Better visualization and less radiation exposure are key tenets in the development of digital subtraction angiography. As the complexity of disease types increases and more technically challenging, cutting-edge procedures are performed, procedure times are prolonged and radiation exposures are increased. Digital subtraction angiography systems with flat panel detectors (FIG. 1) have several theoretical advantages

Address correspondence and reprint requests to: Vallabh Janardhan, M.D., 420 Delaware Street SE, MMC 295, 12-158 PWB, Department of Neurology, University of Minnesota Medical School, Minneapolis, MN 55455. E-mail: vallabh@umn.edu. over digital subtraction angiography systems using the conventional image intensifier television systems, such as high spatial resolution, wide dynamic range, square field of view, and real-time imaging capabilities with no geometric distortion-all of which may be used for improved image quality, reduced patient exposure to radiation, or both. ${ }^{1}$ Hatakeyama et al. ${ }^{1}$ have shown that two-dimensional (2D) and three-dimensional (3D) digital subtraction angiography using the flat panel detector system of the direct conversion type, with low radiation dose, is superior in image quality for visualizing small intracranial vessels with significantly decreased radiation exposure compared with digital subtraction angiography with the conventional image intensifier television system.

Three-dimensional road map. The digital 2D road map technique is routinely used in neurointerventional procedures. The main limitation of the 2D road map technique is that it is a static projection of the vessels on the real time 2D fluoroscopic image; therefore rotation of the $\mathrm{C}$-arm and $\mathrm{X}$-ray equipment will lose the road map and require a repeat contrast injection. With the $3 \mathrm{D}$ road map technique, a 3D rotational angiogram is performed and the $3 \mathrm{D}$ reconstructed image is accurately superimposed on the real time 2D fluoroscopic image using a 3D workstation (FIG. 2). ${ }^{2}$ Therefore, this 3D road map technique has the advantage by using a single injection of contrast medium for the $3 \mathrm{D}$ rotational angiogram in 


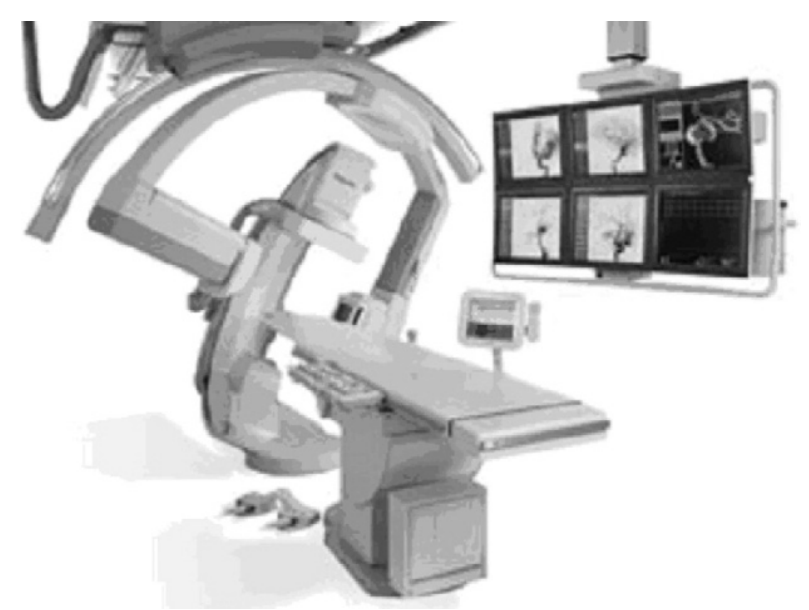

FIG. 1. A biplane three-dimensional rotational digital subtraction angiography system with flat-panel detectors. (Courtesy of Koninklijke Philips Electronics N.V.)

which a $3 \mathrm{D}$ reconstructed image is superimposed onto the real time 2D fluoroscopic image that allows for free positioning of the $\mathrm{C}$-arm and alterations in the distance between the X-ray tube and the image intensifier. This also allows for changes in image magnification without loss of the 3D vessel road map. ${ }^{2}$ The other clinical application of this 3D road map technique is that intracranial procedures (e.g., coil embolization of cerebral aneurysms routinely performed with biplane digital subtraction angiography) can be performed using a single plane digital subtraction angiography system with this 3D road map capability. ${ }^{2}$ The main limitation of this technique for the accurate superimposition of the 3D reconstructed image on the 2D fluoroscopic image is that

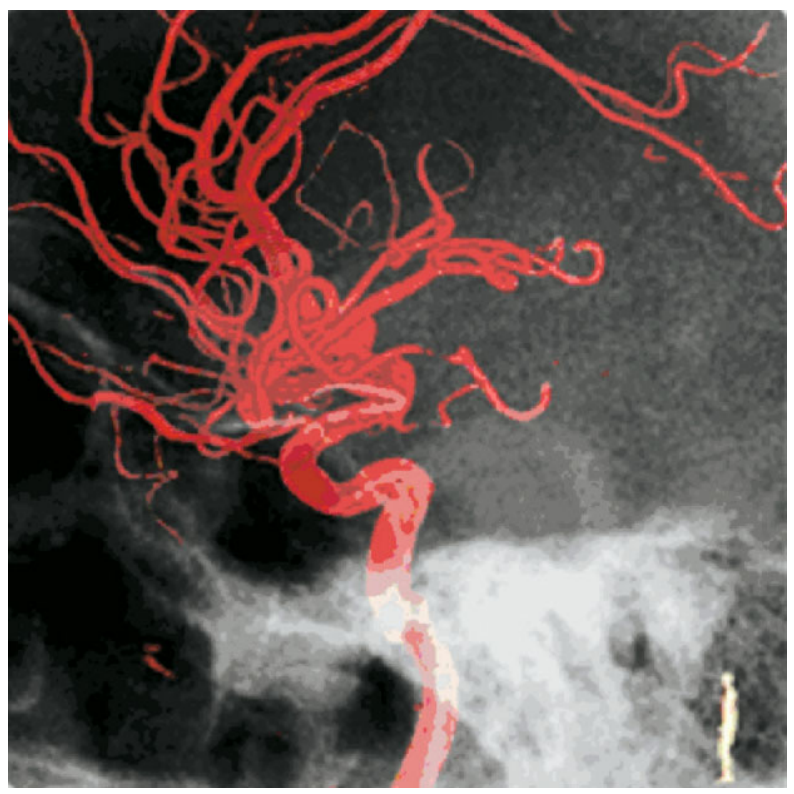

FIG. 2. Three-dimensional (3D) road map with 3D reconstructed image superimposed on two-dimensional fluoroscopic image. (Courtesy: Koninklijke Philips Electronics N.V.)

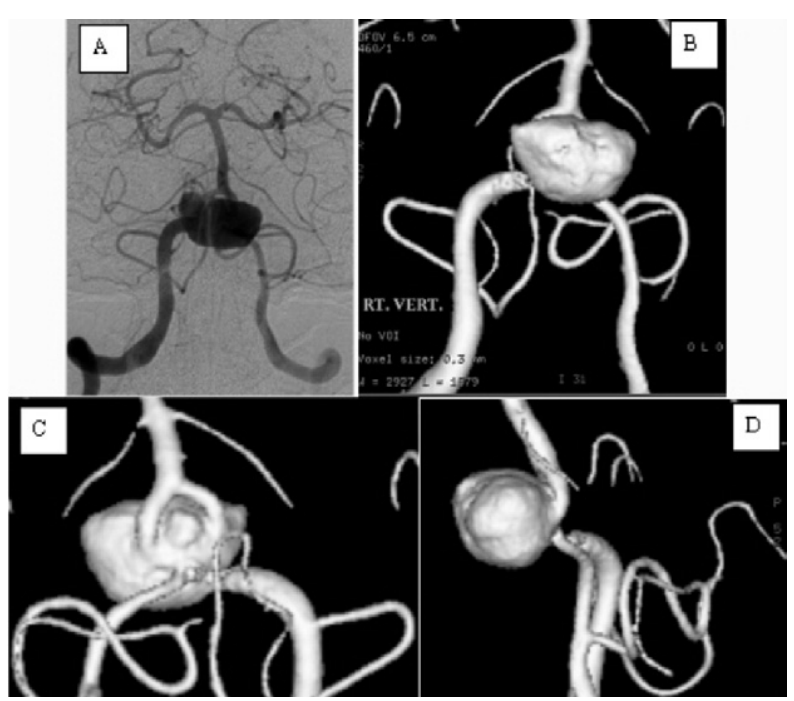

FIG. 3. A: Biplane angiography was performed and intracranial view of the right vertebral artery in the anterio-posterior (AP) projection shows a large vertebro-basilar junction aneurysm. B, C, D: Three-dimensional (3D) rotational angiography was performed and intracranial view of the right vertebral artery in the $3 D$ projections delineates the aneurysm much better than in the AP projection alone. (Courtesy of Yves Pierre Gobin, MD, Division of Interventional Neuroradiology, Departments of Radiology and Neurosurgery, Weill-Medical College of Cornell University, NY.)

the patient's head must remain still, which often requires the patient to be under general anesthesia to avoid motion artifact. $^{2}$

Three-dimensional rotational angiography. Threedimensional rotational angiography is a technical advance in neurointerventional procedures. ${ }^{3}$ Images acquired on the 3D rotational angiogram are readily reconstructed in a 3D workstation within minutes and give a $3 \mathrm{D}$ view of the vessels. This eliminates the need for multiple oblique views, decreases the amount of contrast injected, and decreases the duration of radiation exposure and procedure time. ${ }^{3}$ In addition, in patients with cerebral aneurysms, 3D rotational angiography gives a better delineation of the neck of the aneurysm with respect to the parent vessel, allowing more precise determination of the optimum angle of view for performing the embolization procedure, and it provides an accurate measurement of the size of coils needed for the embolization (FIG. 3). ${ }^{3}$ Three-dimensional rotational angiograms are also helpful in identifying the size of stents needed in steno-occlusive disease. ${ }^{3}$ In addition, 3D reconstruction software is helpful in viewing vessels in a transparent mode that can identify lesions (e.g., vessel injuries) that would not be identified on routine $2 \mathrm{D}$ angiography (FIG. 4). This method also assists in performing angioscopy on the 3D reconstructed image, using a 3D workstation, and aids in the understanding of the true lumen and false lumen in patients with dissections. 


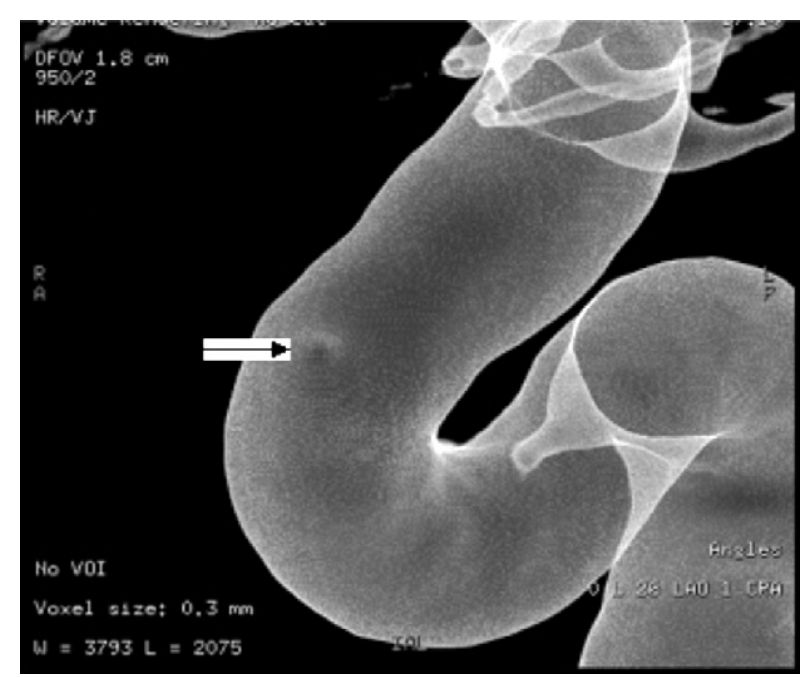

FIG. 4. Intracranial view of the right internal carotid artery in the three-dimensional projection (transparent mode) shows the site of traumatic injury to the internal carotid artery (arrow) that subsequently developed into a pseudoaneurysm at this site. (Courtesy of Howard A. Riina, MD, Division of Interventional Neuroradiology, Departments of Neurosurgery and Radiology, WeillMedical College of Cornell University, NY.)

\section{ULTRASONONOGRAPHY-BASED ADVANCES}

\section{EKOS microcatheter and intra-arterial thrombolysis for ischemic stroke}

Shock wave lithotripsy was first introduced in 1982 and is now routinely used for the treatment of renal and ureteral calculi. ${ }^{4}$ The propagation of shock waves is based on the laws of acoustics and has been shown to help with calcific stone fragmentation. ${ }^{4}$ In vitro studies have also shown that ultrasound waves at $1 \mathrm{MHz}$ can potentate enzymatic fibrinolysis by nonthermal mechanisms in clots treated with recombinant tissue plasminogen activator (r-tpa). ${ }^{5}$ It is speculated that the ultrasonongraphic pulse wave generates local convection currents and micro- streaming causing cavitation, and this, in turn, increases the effective surface area of thrombolytic drug action. ${ }^{6}$ The EKOS MicroLysUS infusion catheter (EKOS Corporation, Bothell, WA) was designed to optimize these principles. This serves as a microcatheter through which microinfusions can be performed via the distal port. This also creates a microenvironment of ultrasonic vibration to facilitate thrombolysis. ${ }^{6}$ Pilot studies have shown the safety and feasibility of this microcatheter in acute ischemic stroke. ${ }^{6}$ The EKOS NeuroWave Catheter (EKOS Corporation) (FIG. 5) is being further studied in a multicenter randomized trial, the Interventional Management of Stroke Study. ${ }^{7}$

\section{Transcranial Doppler ultrasonography and thrombolysis for ischemic stroke}

Transcranial Doppler (TCD) ultrasonography has also been studied as a means of augmenting the action of r-tpa in patients with acute ischemic stroke. ${ }^{8}$ The CLOTBUST-II (Combined Lysis of Thrombus in Brain Ischemia with Transcranial Ultrasound and Systematic Tissue Plasminogen Activator-Phase II) trial evaluated whether transcranial Doppler ultrasound at $2 \mathrm{MHz}$ could safely enhance the thrombolytic activity of r-tpa given systemically. ${ }^{8} \mathrm{~A}$ total of 126 patients were randomly assigned to receive continuous TCD (63 patients) or a placebo (63 patients). ${ }^{8}$ Complete recanalization (determined by TCD monitoring) or dramatic clinical recovery within $2 \mathrm{~h}$ after the administration of an r-tpa bolus occurred in 31 patients in the target group (49\%), compared with 19 patients in the control group $(30 \% ; \mathrm{p}=0.03){ }^{8}$ In patients with acute ischemic stroke, continuous TCD augmented r-tPA-induced arterial recanalization with a nonsignificant trend toward an increased rate of recovery from stroke as compared with placebo. ${ }^{8}$

Microbubbles are small air- or gas-filled microspheres with specific acoustic properties that make them useful as ultrasound contrast agents. ${ }^{9}$ Experimental studies have shown that sonothrombolysis may be further enhanced by the administration of microbubbles. ${ }^{9}$ The application of high-acoustic-pressure ultrasound has been shown to induce nonlinear oscillations of microbubbles, which ultimately lead the microbubbles to explode. Thus, ultrasound-mediated microbubble destruction may further accelerate the clot-dissolving effect of ultrasound. ${ }^{9}$ The synergistic effect of TCD $(2 \mathrm{MHz})$ and microbubbles on sonothrombolysis was evaluated in 111 patients with acute strokes attributable to middle cerebral artery (MCA) occlusion who were treated with intravenous r-tpa. ${ }^{9}$ Thirty-eight patients were treated with r-tpa plus continuous 2-hour TCD monitoring, plus 3 doses of $2.5 \mathrm{~g}$ $(400 \mathrm{mg} / \mathrm{mL})$ of galactose-based microbubbles given at 2, 20, and $40 \mathrm{~min}$ after r-tpa bolus (MB group). These patients were compared with 73 patients who were allo-

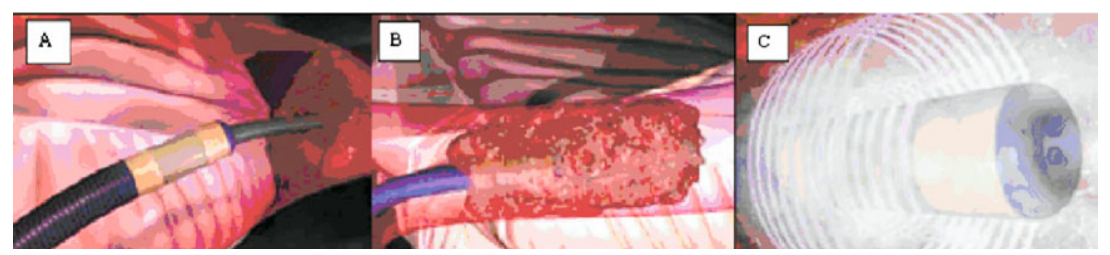

FIG. 5. A: The EKOS NeuroWave Catheter is advanced into the clot over a microwire. B: The microwire is then removed and administration of contrast media via the distal port of the microcatheter can be performed. C: Acoustic streaming of the ultrasound helps disperse the diagnostic agent in areas of low blood flow. (Courtesy of EKOS Corporation, Bothell, WA.) 


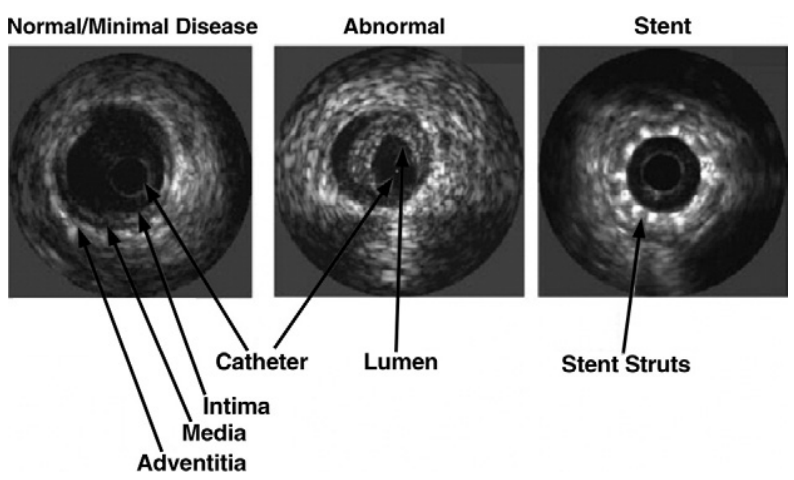

FIG. 6. Intravascular ultrasonography of coronary vessels using the Atlantis SR Pro coronary imaging catheter. (Courtesy of Boston Scientific Corporation, Fremont, CA.)

cated to receive r-tpa plus continuous 2-hour TCD monitoring (r-tpa/TCD group) or r-tpa plus placebo monitoring (r-tpa group). Two-hour complete recanalization rate (determined by TCD monitoring) was significantly higher $(p=0.038)$ in the r-tpa/TCD/MB group (54.5\%) compared with the r-tpa/TCD (40.8\%) and r-tpa (23.9\%) groups. Administration of microbubbles induces further acceleration of ultrasound-enhanced thrombolysis in acute stroke, leading to a more complete recanalization and a trend toward better clinical outcomes. ${ }^{9}$

\section{Intravascular ultrasonography}

Intravascular ultrasonography (IVUS) has been shown to be safe and effective for assessing coronary plaque morphology and evaluating adequate stent deployment (FIG. 6).$^{10}$ Clark et al. ${ }^{10}$ have shown that IVUS is also safe and feasible in patients with carotid artery stenosis prior to carotid angioplasty and stenting. They have shown that IVUS has helped identify superficial lumen calcifications that have been an important predictor of peri-procedural stroke, and they have also shown that IVUS is superior to conventional angiography in assessing stent dimensions, expansion, and apposition. ${ }^{10}$ Further studies are needed to understand the role of IVUS in understanding plaque morphology in patients with carotid artery stenosis.

\section{CT-BASED ADVANCES}

\section{Digital subtraction angiography systems using flat-panel detector technology with combined CT capability}

With advances in interventional neurology procedures, such as recanalization for acute ischemic stroke, embolization for cerebral aneurysms, or brain arteriovenous malformations, it is important to be able to immediately identify complications such as intracranial bleeding. The ability to perform CT scans dynamically during neuroendovascular procedures (thereby avoiding the delay in waiting for the procedure to be completed and transporting the patient to another location for the CT scan) can significantly aid in critical decision making and could be lifesaving. Digital subtraction angiography systems with flat panel detectors have also been developed with combined capability of producing improved cone-beam volume CT images. ${ }^{11}$ Heran et al. ${ }^{11}$ have reported their preliminary findings supporting the usefulness of such a combined system, wherein 3D rotational digital subtraction angiography or cone-beam volume CT can be obtained interchangeably with the same flat-panel detector $\mathrm{C}$-arm motion without patient transfer.

\section{4-slice CT}

The development of 64-slice CT scanners has significantly improved the resolution of CT angiography of the coronary vessels as compared with conventional angiography. ${ }^{12}$ Experience at our center is also similar with regard to CT angiography of the extracranial and intracranial vessels (FIG. 7), and CT perfusion of the brain using 64-slice CT (FIG. 8).

\section{MRI-BASED ADVANCES}

\section{MR-guided endovascular interventions}

Currently X-rays are used for visualizing endovascular catheter interventions. Magnetic resonance imaging has better anatomic delineation of tissues and can serve as an alternative or adjunctive modality for guiding endovas-

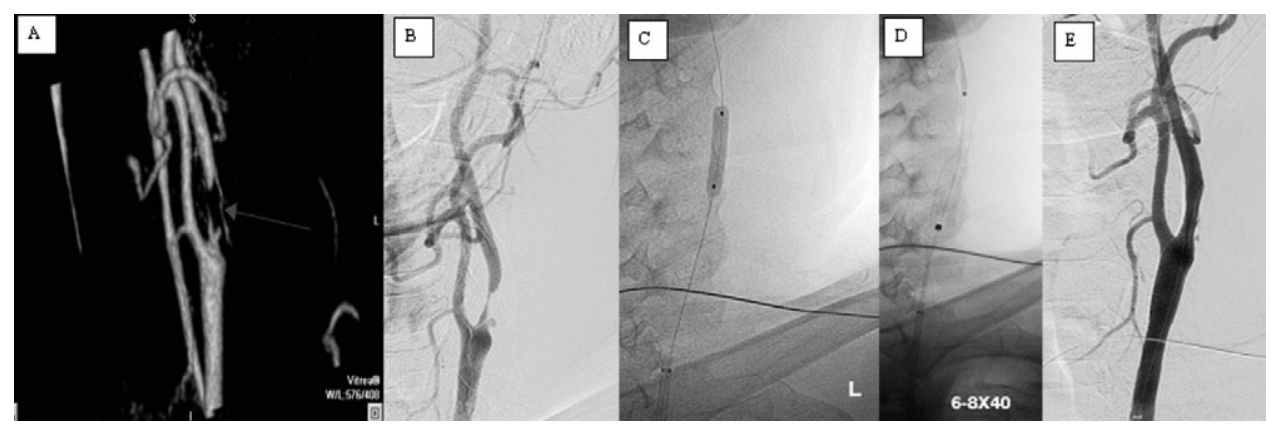

FIG. 7. A: Computed tomographic (CT) angiography showing the critical stenosis in the origin of the left internal carotid artery in a 46-year-old man using a 64-slice CT scanner (B) compared with the conventional angiogram showing the similar stenosis. After carefully deploying a distal embolic protection device, (C) balloon angioplasty and (D) stenting were performed. E: Subsequently, the distal protection device was carefully removed and final angiogram showing marked improvement post-treatment. 


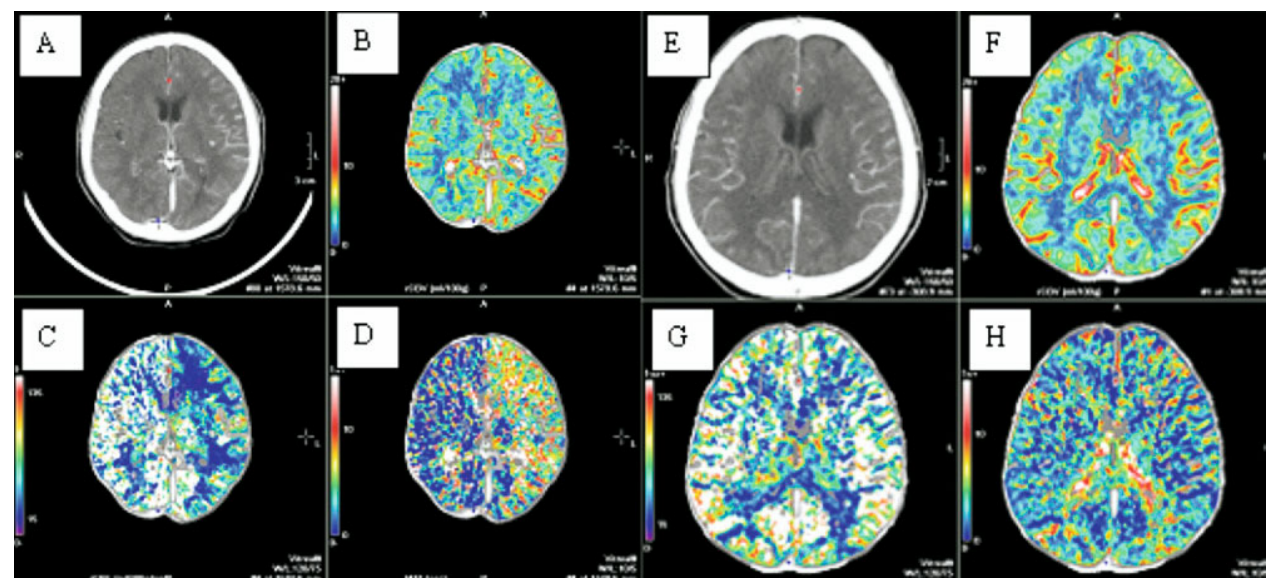

FIG. 8. A-D: Computed tomographic (CT) perfusion of the brain using the 64-slice CT scanner in a 46-year-old male patient with critical symptomatic left internal carotid artery (ICA) stenosis pretreatment, (C) showing marked decrease in regional cerebral blood flow, (D) delayed mean transit time, (A) with no infarct on noncontrast head CT, (B) and preserved regional cerebral blood volume on the left ICA territory. (E-H)Computed tomographic perfusion of the brain using the 64-slice CT scanner in the patient with critical symptomatic left ICA stenosis postballoon angioplasty and stenting, (G) showing marked improvement in regional cerebral blood flow, (H) normalized mean transit time, (E) with no infarct on noncontrast head CT, and (F) preserved regional cerebral blood volume on the left ICA territory.

cular interventions. ${ }^{13}$ Experimental studies have shown that tracking catheters using MRI is possible. ${ }^{14}$ Some of the limitations of MR-guided endovascular intervention include the lack of clinical grade devices that are MR compatible and the prohibitive cost for transition from an $\mathrm{X}$-ray to an MR-guided endovascular intervention system. Future research with improved algorithms for active and passive tracking of catheters, stents, and other devices, and a combination of MR and X-ray visualization techniques might make MR-guided neurointerventional procedures a reality. ${ }^{13}$

\section{Intravascular MRI}

Intravascular ultrasonography has been shown to be useful in evaluating the lumen of atherosclerotic lesions in coronary and carotid vessels and also in aiding optimal stent deployment. ${ }^{10}$ However, there are limitations with the luminal visualization obtained with intravascular ultrasonography. Magnetic resonance imaging is superior to ultrasonography in defining anatomic details of tissues. Although surface MRI is useful in studying atherosclerotic plaques in superficial arteries, deep arteries are difficult to visualize. ${ }^{15}$ Experimental studies have shown that intravascular MRI is a useful tool to study human atherosclerotic plaque morphology in the iliac arteries. ${ }^{15}$ Future studies need to evaluate this technique in extracranial and intracranial arterial territories.

\section{MOLECULAR NEUROIMAGING ADVANCES}

\section{Lipid microbubbles for imaging of inflamed plaque} and cell surface receptor imaging

Microbubbles coated with albumin or lipid shells tend to bind to tissue leukocytes and this binding is dependent on leukocyte activation and expression of adhesion molecules in regions of inflammation. ${ }^{16}$ After attachment of micro- bubbles to leukocytes, microbubbles may be phagocytosed intact by neutrophils and monocytes, but they continue to oscillate and generate an ultrasound contrast effect. ${ }^{16}$ The interactions between microbubbles and activated leukocytes have provided a means to image inflammation with ultrasound. ${ }^{16}$ In the coronary arteries, intravascular ultrasonography has been useful in identifying these microbubbles bound to plaque and serves as a molecular imaging tool to identify the vulnerable plaque. ${ }^{17}$ The role of microbubbles in identifying inflamed plaque in the carotid arteries using intravascular ultrasonography is currently being studied at our center. These microbubbles can also be conjugated with monoclonal antibodies to specific cell surface receptors and therefore can serve as a molecular and cellular imaging tool. $^{16}$

\section{CONCLUSIONS}

Advances in neuroimaging modalities such as digital subtraction angiography, ultrasonography, CT, MRI, and molecular imaging have dramatically advanced the field of interventional neurology and neuroimaging.

\section{REFERENCES}

1. Hatakeyama Y, Kakeda S, Korogi Y, et al. Intracranial 2D and 3D DSA with flat panel detector of the direct conversion type: initial experience. Eur Radiol 2006;16:2594-2602.

2. Soderman M, Babic D, Homan R, Andersson T. 3D roadmap in neuroangiography: technique and clinical interest. Neuroradiology 2005;47:735-740.

3. Anxionnat R, Bracard S, Macho J, et al. 3D angiography. Clinical interest. First applications in interventional neuroradiology. J Neuroradiol 1998;25:251-262.

4. Auge BK, Preminger GM. Update on shock wave lithotripsy technology. Curr Opin Urol 2002;12:287-290.

5. Francis CW, Onundarson PT, Carstensen EL, et al. Enhancement of fibrinolysis in vitro by ultrasound. J Clin Invest 1992;90:20632068. 
6. Mahon BR, Nesbit GM, Barnwell SL, et al. North American clinical experience with the EKOS MicroLysUS infusion catheter for the treatment of embolic stroke. AJNR Am J Neuroradiol 2003; 24:534-538.

7. The Internet Stroke Center: Stroke trials registry. Available at: www.strokecenter.org/trials/browse.asp?browse $=$ acute. Last accessed: March 14, 2007.

8. Alexandrov AV, Molina CA, Grotta JC, et al. CLOTBUST Investigators. Ultrasound-enhanced systemic thrombolysis for acute ischemic stroke. N Engl J Med 2004;351:2170-2178.

9. Molina CA, Ribo M, Rubiera M, et al. Microbubble administration accelerates clot lysis during continuous 2-MHz ultrasound monitoring in stroke patients treated with intravenous tissue plasminogen activator. Stroke 2006;37:425-429.

10. Clark DJ, Lessio S, O’Donoghue M, Schainfeld R, Rosenfield K. Safety and utility of intravascular ultrasound-guided carotid artery stenting. Catheter Cardiovasc Interv 2004;63:355-362.

11. Heran NS, Song JK, Namba K, Smith W, Niimi Y, Berenstein A. The utility of DynaCT in neuroendovascular procedures. AJNR Am J Neuroradiol 2006;27:330-332.
12. Pugliese F, Mollet NR, Runza G, et al. Diagnostic accuracy of non-invasive 64-slice CT coronary angiography in patients with stable angina pectoris. Eur Radiol 2006;16:575-582.

13. Rhode KS, Sermesant M, Brogan D, et al. A system for real-time XMR guided cardiovascular intervention. IEEE Trans Med Imaging 2005;24:1428-1440.

14. Wacker FK, Elgort D, Hillenbrand CM, Duerk JL, Lewin JS. The catheter-driven MRI scanner: a new approach to intravascular catheter tracking and imaging-parameter adjustment for interventional MRI. AJNR Am J Neuroradiol 2004;183:391-395.

15. Larose E, Yeghiazarians Y, Libby $P$, et al. Characterization of human atherosclerotic plaques by intravascular magnetic resonance imaging. Circulation 2005;112:2324-2331.

16. Lindner JR. Evolving applications for contrast ultrasound. Am J Cardiol 2002;90:72J-80J.

17. Vavuranakis M, Kakadiaris IA, O’Malley SM, et al. Images in cardiovascular medicine. Detection of luminal-intimal border and coronary wall enhancement in intravascular ultrasound imaging after injection of microbubbles and simultaneous sonication with transthoracic echocardiography. Circulation 2005;112:e1-2. 